\title{
Surfactants: physicochemical interactions with biological macromolecules
}

\author{
M. Aguirre-Ramírez • H. Silva-Jiménez • I. M. Banat • M. A. Díaz De Rienzo
}

Received: 24 January 2020/Accepted: 7 December 2020/Published online: 3 February 2021

(C) The Author(s) 2021

\begin{abstract}
Macromolecules are essential cellular components in biological systems responsible for performing a large number of functions that are necessary for growth and perseverance of living organisms. Proteins, lipids and carbohydrates are three major classes of biological macromolecules. To predict the structure, function, and behaviour of any cluster of macromolecules, it is necessary to understand the interaction between them and other components through basic principles of chemistry and physics. An important number of macromolecules are
\end{abstract}

M. Aguirre-Ramírez and H. Silva-Jiménez equally contributed to this work.

\section{Aguirre-Ramírez}

Departamento de Ciencias Químico Biológicas, Instituto de Ciencias Biomédicas, Universidad Autónoma de

Ciudad Juárez, Ciudad Juárez, Chihuahua, Mexico

\section{H. Silva-Jiménez}

Área de Oceanografía Química, Instituto de Investigaciones Oceanológicas, Universidad Autónoma de Baja California, Ensenada, Baja California, Mexico

I. M. Banat

School of Biomedical Sciences, University of Ulster, Coleraine BT52 1SA, Northern Ireland, UK

M. A. Díaz De Rienzo ( $₫)$

School of Pharmacy and Biomolecular Sciences, Liverpool John Moores University, James Parsons Building 10.05C, Byrom Street, Liverpool L3 3AF, UK e-mail: m.a.diaz@ljmu.ac.uk present in mixtures with surfactants, where a combination of hydrophobic and electrostatic interactions is responsible for the specific properties of any solution. It has been demonstrated that surfactants can help the formation of helices in some proteins thereby promoting protein structure formation. On the other hand, there is extensive research towards the use of surfactants to solubilize drugs and pharmaceuticals; therefore, it is evident that the interaction between surfactants with macromolecules is important for many applications which includes environmental processes and the pharmaceutical industry. In this review, we describe the properties of different types of surfactants that are relevant for their physicochemical interactions with biological macromolecules, from macromolecules-surfactant complexes to hydrophobic and electrostatic interactions.

Keywords Surfactants - Macromolecules · Biological systems $\cdot$ Molecular interactions

\section{List of symbols \\ $\gamma_{11}$ Surface tension when two identical phases are considered \\ $\gamma_{1} \quad$ Surface tension of phase 1 i.e. liquid \\ $\gamma_{2} \quad$ Surface tension of phase 2 i.e. solid \\ $\gamma_{12}$ Interfacial tension between two different phases \\ $w_{11}$ Work of adhesion between two identical phases \\ $w_{12}$ Work of adhesion between two different phases}




\section{Introduction}

Surfactants are amphiphilic molecules capable of reducing the surface tension between two immiscible phases (Otzen 2017). These molecules are either chemically produced (synthetic surfactants) or based on biological materials (biosurfactants). The reduction of surface tension is due to their amphiphilic properties, as their molecules consist of both hydrophilic and hydrophobic moieties (Li and Lee 2019). The hydrophilic part contains heteroatoms such as oxygen, sulphur, nitrogen and phosphorous, which appear in functional groups such as alcohol, thiol, ether, ester, acid, sulphate, sulfonate, phosphate, amine, amide, etc., while the hydrophobic part is typically a paraffin, cycloparaffin or aromatic hydrocarbon, which may contain halogens. Due to their dual affinity, amphiphilic molecules are not stable either in polar or in organic solvents. To meet both types of affinities, the hydrophilic moiety must be surrounded by a polar solvent, while the hydrophobic moiety must be in contact with an organic solvent. Such conditions exist only between two immiscible phases. The boundary between a condensed phase and a gaseous phase is referred to as a surface, and the boundary between two condensed phases such as two liquids or a liquid and a solid, is referred to as an interphase. Many properties of surfactants depend on this strong affinity for surfaces or interphases (Khan et al. 2015).

There are important properties that characterise each particular system. Surface tension is defined as the work required to increase the area of a surface isothermally and reversibly by unit amount (Ebnesajjad 2014). Surface tension $(\gamma)$ is expressed as surface energy per unit area and alternatively as a force per unit length. If we consider two identical phases the surface tension $\left(\gamma_{1}\right)$ can be expressed by Eq. 1:

$\gamma_{1}=\frac{1}{2} w_{11}$

where $W_{11}$ represents the work of adhesion between the two identical phases, which is defined as the reversible thermodynamic work required to separate the interface from the equilibrium state of the two phases to a separation distance of infinity.

On the other hand, the interfacial tension between two different phases ( 1 and 2 ) can be given by Eq. 2:

$\gamma_{12}=\gamma_{1}+\gamma_{2}-w_{12}$
These characteristics are determinant in terms of the properties of the systems, such as the existence and persistence of emulsions or foams, where surfactants are responsible for the changes (reduction) in surface tension. Surfactants allow the mixing of hydrophilic molecules with hydrophobic ones, through the formation of structures called micelles which allow the association of both types of molecules in a single phase. This compatibility between molecules that do not have a natural affinity is also known as cosolubilisation (Poša et al. 2019) and can be used to establish different applications.

Surfactants are used in a wide range of industrial applications (Banat and Thavasi 2018). In agriculture, for example, phytosanitary agents are applied in the form of aerosol (surfactant) which, sometimes, contains a dispersed organic phase (emulsifier) to dissolve herbicides and insecticides (Marquez et al. 2018). While in food products, they contribute to the conditioning of creams, suspensions, emulsions, soluble or dispersible powders (Kralova and Sjöblom 2009). In mining processes, they play an important role in the flotation and leaching of metals like iron, zinc, uranium (Asselin and Ingram 2014; Diaz et al. 2015); as well as in the textile industry to improve the performance of different operations and to provide particular properties to the finished products (Pacifico and Giers 1995; Proffitt and Patterson 1988). In the oil industry, they have been used to help to solve problems caused by drilling operations to the conditioning of the finished products; in fact, extracted crude oil reaches the surface in the form of a water-inoil emulsion, which makes it essential to remove or separate the water content (Marquez et al. 2019).

Chemical surfactants are derived from nonbiodegradable components, and in some cases can cause serious problems to the environment, such as: (1) the formation of foams which inhibit or paralyze natural (or artificial) purification processes, concentrate impurities and can spread bacteria or viruses; (2) the increase of phosphate content in basins, from polyphosphates that are used in combination with surfactants (Santos et al. 2016).

Given the problems caused by synthetic surfactants, different studies have been carried out over the past years, seeking to find alternative products compatible with the environment and have demonstrated the feasibility of producing these compounds from 
microorganisms (Akbari et al. 2018). Most microbial biosurfactants are typically biodegradable, biocompatible and have stable activities under extreme environmental conditions (Naughton et al. 2019). Hence the interest to study their production from fungi and bacteria, among which the genera Bacillus and Pseudomonas stand out. Many of these biosurfactants produced by Pseudomonas aeruginosa have been characterized and studied as agents capable of removing hydrophobic compounds from soil (Geetha et al. 2018), antimicrobials and biofilm disruptors (Elshikh et al. 2017; Diaz De Rienzo et al. 2016; Ceresa et al. 2020). Although the physicochemical properties of (bio) surfactants have been well documented through the years (Mankowich 1953; Behrens 1964; Van Os et al. 1993; Patino et al. 2007; Morais et al. 2017), their interaction with biological components has had less focus. This review therefore focuses on the properties of surfactants that are relevant for their physicochemical interactions with biological systems (Fig. 1), and when possible compare them with their biological counterparts.

\section{Surfactant-protein interactions}

The study of the interactions between surfactants, both synthetic and microbial (biosurfactants), with proteins is of great interest in various biotechnology fields and industries such as food, cosmetics, pharmaceutical, biomedical, and environmental (Lee et al. 2011; Otzen 2011; Tucker et al. 2014; Malik 2015). In the biomedical industry, protein-surfactant systems are used for the production of hydrogels (Afinjuomo et al. 2019; Castelli et al. 2008). The hydrogels form the base of fibrous proteins such as fibroin, which are used for tissue regeneration and drug delivery (Park et al. 2014; Dubey et al. 2018; Ohadi et al. 2020).

There are three main forces that drive the proteinsurfactant interaction: (1) electrostatic, (2) hydrophobic and (3) Van der Waals (Mackie and Wilde 2005; Li and Lee 2019). The dominant interaction is determined by the nature of both molecules and their concentration (Mehan et al. 2015; Li and Lee 2019). These molecular interactions have an influence on the native structure of proteins promoting or preventing denaturation, aggregation and loss of enzymatic activity among other factors (Mehan et al. 2015). Surfactants of biological origin have an advantage over synthetic surfactants in terms of their ability to prevent denaturation of proteins and a reduction in their aggregation (Otzen 2011, 2017).

The protein-surfactant systems mainly studied are those that contain globular proteins such as bovine serum albumin (BSA), $\alpha$-lactoglobulin and $\beta$-glucosidase. In contrast, very few studies have been performed exploring the fibrous protein-surfactant systems. Type I collagen, silk fibroin, and keratin are fibrous proteins that have been studied in combination with ionic and non-ionic surfactants (Maldonado et al. 1991; Mandal and Kund 2008; Kezwon et al. 2016; Kezwoń and Wojciechowski 2016; Pan et al. 2016; Park et al. 2014; Dubey et al. 2018). A few studies suggest that the molecular interactions presented by fibrous proteins (collagen, fibroin, keratin) in combination with ionic and non-ionic surfactants are similar to the globular protein-surfactant systems (Lee et al. 2011; Khan et al. 2015, Kezwon et al. 2016; Kezwoń and Wojciechowski 2016; Pan et al. 2016).

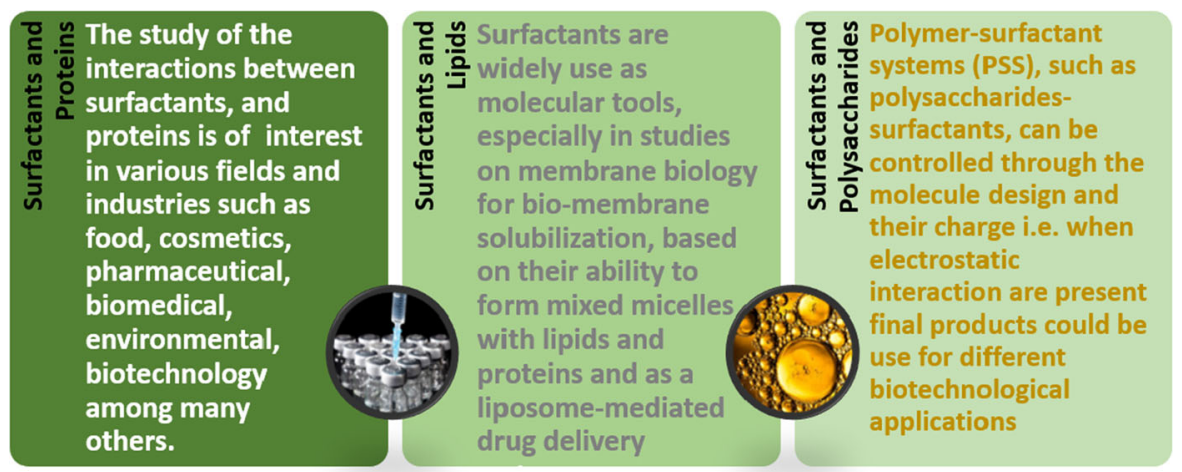

Fig. 1 Illustrative summary of the main types of interactions between (bio)surfactants and macromolecules 
Type I collagen interacts with Sodium Dodecyl Sulphate (SDS), Cetyl Trimethyl Ammonium Bromide (CTAB), and Triton X-100 through hydrophobic and electrostatic molecular interactions. The predominance of a particular molecular interaction depends on the type of surfactant, i.e. surfactants could produce changes in collagen secondary structure (Maldonado et al. 1991; Kezwon et al. 2016; Kezwoń and Wojciechowski 2016).

The main physical parameters that have an effect on the surfactant-protein interactions are: (a) the surfactant concentration; (b) the chemical nature of surfactant (ionic or non-ionic surfactants); and (c) the secondary structure of the protein ( $\alpha$-helix and $\beta$ sheets) (Díaz et al. 2003; Malik 2015).

\section{Surfactant concentration}

The effect on stabilization or destabilization mediated by a surfactant is dependent on the concentration of the surfactant (Mehan et al. 2015). In that sense, many surfactants (biological and synthetic ones), usually promote protein stabilization at concentrations far below Critical Micelle Concentration (CMC), while at concentrations higher than the $\mathrm{CMC}$ there is an opposite effect, they promote denaturation, aggregation, as well as loss of biological function of proteins (Díaz et al. 2003; Otzen 2011; Malik 2015). In general, the binding of the surfactant to the protein is carried out in three phases. In the binding phase (phase I), individual surfactant molecules bind to the protein without causing any structural change, and electrostatic interactions dominate over hydrophobic ones. In the cooperative phase (phase II), the increase in the surfactant concentration reaches a sub-CMC levels, triggering the formation of the hydrophobic clusters that start to bind to the hydrophobic regions of proteins leading to their denaturation and changes in the secondary structure. In this phase, hydrophobic interactions dominate over electrostatic; in addition, the unfolding process increases linearly (Otzen 2011; Malik 2015). Finally, the saturation phase (phase III) is where the protein binding sites are already saturated. In this phase, there are free surfactant molecules that interact with the protein-bound micelles and no longer cause further changes (Malik 2015).
Chemical nature of surfactants

Surfactants can be divided into two groups according to their chemical composition: ionic and non-ionic. The ionic surfactants, according to their charge, can be anionic or cationic (Otzen 2011; Khan et al. 2015). The hydrophilic group of the surfactant affects the stability of the protein because it can tightly bind to the protein causing its denaturation and contributes to the solubilization of the membrane proteins (Mehan et al. 2015). Anionic surfactants are typically protein-denaturing agents (Khan et al. 2015). Among the anionic surfactants, SDS is well known for having strong electrostatic interactions with proteins (Deep and Ahluwalia 2001; Otzen et al. 2009; Hansted et al. 2011; Otzen 2011). These interactions are generated between the positively charged amino acids present in the primary structure of the protein along with the interactions of the hydrocarbon chains of the surfactant, and the aliphatic regions of the amino acids arginine (Arg) and lysine (Lys) (Otzen et al. 2009).

Such properties have been used in some protein separation and/or solubilisation techniques. The interaction between SDS and several globular proteins has been previously reported, i.e. the denaturing effect of SDS on $\alpha$-lactalbumin occurs in different stages depending on the concentration of the surfactant. In the early stages, SDS monomers bind to the protein to form groups up to a critical concentration that results in the start of the denaturation process (Fig. 2). The binding of more monomers results in the loss of the secondary structure of the protein (Otzen et al. 2009). In the case of $\beta$-lactoglobulin, SDS has an opposite effect to the one observed with $\alpha$-lactalbumin, since this amphiphilic molecule reduces the aggregation of the protein at concentrations well below its CMC (Hansted et al. 2011).

Compared to anionic surfactants, cationic surfactants have a milder protein destabilization effect (Khan et al. 2015). These ionic surfactants interact with amino acids whose side chains are usually negatively charged like aspartate (Asp) and glutamate (Glu) (Otzen 2011). For example, Khan et al. (2019) reported that the interactions between CTAB and Hen egg white lysozyme are very hydrophobic and weakly electrostatic, which do not cause a change in the secondary structure of the protein but do cause a negative effect on the tertiary structure. 


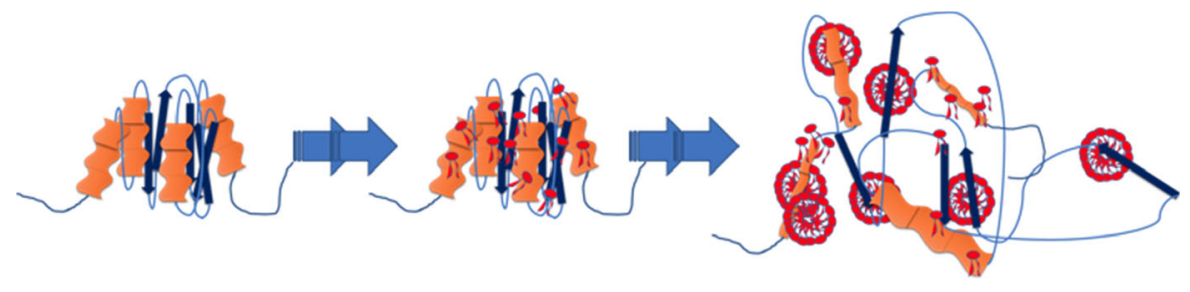

Fig. 2 Representative scheme of the denaturation effect promoted by SDS over $\alpha$-lactalbumin. SDS monomers bind to the protein starting the denaturation process; at a high concentration of SDS monomers, the secondary structure of the protein is lost

In the case of non-ionic surfactants (i.e., dodecyl maltoside, polysorbates), they commonly minimize or prevent protein aggregation (Lee et al. 2011; Otzen 2011). According to various studies, the molecular interactions between proteins and non-ionic surfactants are very weak and the union of the biomolecule with the non-ionic surfactant is driven by hydrophobic interactions, which results in a tendency to solubilize proteins. These surfactants are used in the food industry and have biomedical applications in drug formulations (Lee et al. 2011; Campos et al. 2013; Tucker et al. 2014). Non-ionic surfactants usually have ethoxylate groups that interact with the hydrophobic moieties of proteins, exposing the hydrophilic groups present in both molecules, which results in the increase of the hydrophilicity of the nonionic surfactant-protein complex, thereby reducing the aggregation of proteins (Rudolph and Jones 2002; Ruiz-Peña et al. 2010; Lee et al. 2011; Tucker et al. 2014). The chemical structure of this type of surfactant plays an important role in promoting or preventing protein denaturation, even if the structural differences are minor. Tween type surfactants (ethoxylated polysorbates) vary in the length of the fatty acid hydrocarbon chain and interact differently with BSA, as seen in the number of surfactant molecules that are able to bind to the protein as well as the type of binding (Ruiz-Peña et al. 2010).

Another type of surfactants known as dimeric or Gemini surfactants are constructed of two monomers of surfactants which are joined by a spacer close to the hydrophilic heads (Sinha et al. 2016). Despite their importance in several industrial fields, studies of Protein-Gemini surfactants interactions are limited, compared with those conducted with single chain surfactants (Sinha et al. 2016; Parray et al. 2018; Akram et al. 2019). Several studies have revealed that some interaction mechanisms of these new generation of surfactants with proteins are shared with their corresponding monomers differing in the effects that they induce in the biomolecule, ranging from having stronger molecular interactions than their monomeric counterpart to changes or stabilization in the secondary and tertiary structures of proteins (Sinha et al. 2016; Sonu et al. 2017; Akram et al. 2019). Comparative studies of the interaction of BSA with the cationic surfactant Dodecyl Trimethyl Ammonium Bromide (DTAB) and with three Gemini-surfactants of the bis(dimethyldodecylammonium bromide) family; butanediyl-1,4-bis(dimethyldodecylammonium bromide (12-4-12,2Br -), 2-butanol-1,4bis(dimethyldodecylammonium bromide) (12-4(OH)-12,2Br - ), 2,4-dibutanol-1,4bis(dimethyldodecylammonium bromide) (12$4(\mathrm{OH}) 2-12,2 \mathrm{Br}-)$, showed that at lower concentrations of the surfactant the interaction in the surfactantprotein complex is managed by electrostatic forces and while the concentration of the surfactant increases.

The union of the protein with the surfactant is hydrophobic in nature, which is stronger with the Gemini-surfactant, causing greater denaturation of BSA compared to DTAB, which suggests that the spacer between the two monomers plays an important role (Sinha et al. 2016). Sonu et al. (2017) conducted a study on the effect of surfactant spacers [12-8-12, 2Br], [12-4-12, 2Br-] and [12-4 (OH) -12, 2Br-] on the interaction with $\mathrm{BSA}$ and reported that the more hydrophobic the spacer is, the lower is the reduction in the number of $\alpha$-helices and denaturing effects. Akram et al. (2019) on the other hand, analysed the interaction of the BSA model protein with three members of a family of Gemini Cm-E20-Cm surfactants and demonstrated that the binding of these dimeric surfactants with the protein is considerably strong, without causing a significant loss of $\alpha$-helix (3-4\%), keeping the secondary and tertiary structure of the BSA virtually intact. Other authors have reported that the effect caused by these Gemini-surfactants on the 
various model proteins may be subject to changes at different temperatures, $\mathrm{pH}$ concentrations, ionic strength, and surfactant concentrations, among others (Faustino et al. 2009).

\section{Secondary structure of proteins}

In some cases, the secondary structure of a protein could have an effect on the ability of a surfactant to promote its aggregation or denaturation activities, without necessarily being a specific surfactant-protein interaction. Zaragoza et al. (2012) showed that when the trehalolipid biosurfactant produced by a Rhodococcus sp. is present at a concentration lower than CMC, proteins with a high content of $\alpha$-helix in the secondary structure such as BSA and cytochrome $c$ (Cyt- $c$ ) showed resistance to thermal unfolding and there was no alteration of the secondary structure. In addition, Isothermal Titration Calorimetry (ITC) investigations demonstrated that the interactions between trehalolipids and both proteins are not specific, suggesting the involvement of hydrophobic domains of proteins (Zaragoza et al. 2012). However, the biosurfactant mannosylerythritol lipid-A (MEL-A) has a different influence on the enzyme $\beta$-glucosidase. At CMC values, this biosurfactant promotes a secondary structure changes of $\beta$-glucosidase, causing a decrease in $\beta$-sheets content and an increase in $\alpha$ helices, $\beta$-turn, and random coil. These structural changes cause $\beta$-glucosidase to acquire thermal stability by increasing its midpoint temperature $\left(T_{m}\right)$ and unfolding enthalpy (Fan et al. 2018).

The above can be explained in thermodynamic and structural terms. On the one hand, at CMC values, MEL-A forms micelles, thereby increasing hydrophobic interactions. Thermodynamic data obtained by ITC, support the hypothesis that weak hydrophobic interactions are responsible for the union of MEL-A and $\beta$-glucosidase. On the other hand, the stability gained by $\beta$-glucosidase at CMC values can be given by the enzyme's secondary structural changes. The increase of $\alpha$-helix content is a potential factor which promotes, (1) the exposure of hydrophobic regions to amino acid residues that interact hydrophobically, (2) hydrogen bond formation with fatty acid chains, and (3) hydroxyl groups of glycosidic residues (Otzen 2011; Fan et al. 2018).

Based on various analytical methods, Zhang and $\mathrm{Li}$ (2018) reported that surfactin, a biosurfactant of the lipopeptide type, induces changes in the conformations of the alkaline protease secreted by Bacillus sp., which results in weak hydrophobic interactions, hydrogen bonds and some electrostatic interactions. In addition, they found that the enzymatic activity of the alkaline protease may be affected positively or negatively at low or high concentrations of surfactin, respectively. In the first case, the low concentration of surfactin in the aqueous medium, allows the biosurfactant molecule to interact with the alkaline protease as a cofactor, thus causing an increase in enzymatic activity, while at high concentrations of surfactin, a decrease in enzymatic activity occurs. This is because the hydrophobicity of the alkaline protease is decreased by the high concentration of biosurfactant molecules present in the solution. Finally, the cases analysed in this review on the interactions between different surfactants with a model protein reveal that they are quite diverse, where the physicochemical characteristics of the interacting molecules play an essential role. Molecular interaction studies using various biophysical techniques, will allow us to understand the basis of interaction between surfactants and proteins.

\section{Surfactant-lipid interactions}

The phase behaviour between surfactants-water and lipid-water is well documented (Chernik 2000; Koynova and Tenchov 2001; Ebnesajjad 2006), however the interaction between surfactants and lipids is not well reported with most studies have been carried out on temperature and enthalpy variables without a detailed description of the mechanisms involved (Koynova and Tenchov 2001). Surfactants are widely used as molecular tools, especially in studies of membrane biology for biomembrane solubilization, based on their ability to form mixed micelles with lipids and proteins (Koynova and Tenchov 2001) and as a liposome-mediated drug delivery system (Bnyan et al. 2018). Liposomes have been used as a model of biological membranes for a long time, due to their phospholipid structure. The structure of phospholipids has a hydrophilic head group and a hydrophobic tail group. When dispersed in an aqueous solution, the head is attracted by water, and the tail, including a long hydrocarbon chain, is repelled by water promoting the 
formation of vesicles (Stryer 1981; Dua et al. 2012; Gunay and Ozer 2018).

The interaction between lipids and surfactants is derived in a different numbers of model systems (Helenius and Simons 1975; Lichtenberg et al. 1983). All these models show a general scheme for the interaction between lipids and surfactants (which displays the transition from vesicles to mixed micelles) and is described as a three-stage model (Fig. 3). The first stage is where the surfactant partition between the lipid bilayers and the aqueous phase and start reaching a level where the bilayers break into micelles; the second phase is where there is a mix between micelles and bilayers in a co-existent state and the last phase is characterized by an increase of the surfactant concentration leading to a phase where all the bilayers are solubilized and only lipidrich micelles are present (Lichtenberg et al. 2013; Pizzirusso et al. 2017).

There are different studies that show the three-stage model applied to biological membranes, including homogenous phospholipids systems (phosphatidylcholine and phosphatidylserine), $\mathrm{Ca}^{2+}$-ATPase membranes (Le Maire et al. 2000) and liposomes prepared from SR lipid (Langner and Hui 2000). The solubilisation of membranes generally occurs via the uptake of non-micellar surfactants monomers, which is why when a surfactant is added to solubilize a membrane preparation, if the surfactant concentration is below their CMC, then it is just the monomer fraction that interact with the biological membrane.

When it comes to the study of biosurfactants and membrane lipids interactions, few studies have been reported on molecular interactions (Ortiz et al. 2009; Aranda et al. 2007; Rodrigues et al. 2006; Malaspina et al. 2017). The effect of trehalose lipids on membrane phospholipids was reported by Ortiz et al. (2008) showing that the biosurfactants exhibit a dehydrating effect on the interfacial region of saturated phosphatidylethanolamines promoting the formation of unsaturated phosphatidylethanolamines. The same research group evaluated the effect of trehalose lipid produced by Rhodococcus sp. on the structural properties of dimyristoyl phosphatidylserine (DMPS) membranes. They have showed that the biosurfactant incorporates into the DMPS membranes and increases the fluidity of the phosphatidylserine acyl chains making changes in the environment of the polar head group and, as a consequence, decreases the interfacial tension of the membrane, thereby decreasing the motional freedom of the phospholipids (Ortiz et al. 2009).

One of the most studied biosurfactant in terms of their effect on the plasma membrane is the Iturin produced by Bacillus subtilis. Iturin is an effective antifungal compound and its mechanisms of action is related to the disruption of the biological membrane by the formation of small vesicles and their aggregation in yeast cells (Peypoux et al. 1994; Rodrigues et al. 2006). Iturin was shown to pass through the cell wall and disrupt the plasma membrane with the formation of small vesicles and the aggregation of intramembranous particles, interacting with the nuclear membrane and probably with membranes of other cytoplasmic organelles affecting the morphology and membrane structure of yeast cells (Thimon et al. 1995). Recently, the studies in molecular surfactantlike peptides and lipids has become more focused and significant due to their excellent properties, such as versatility, biocompatibility and medicinal properties

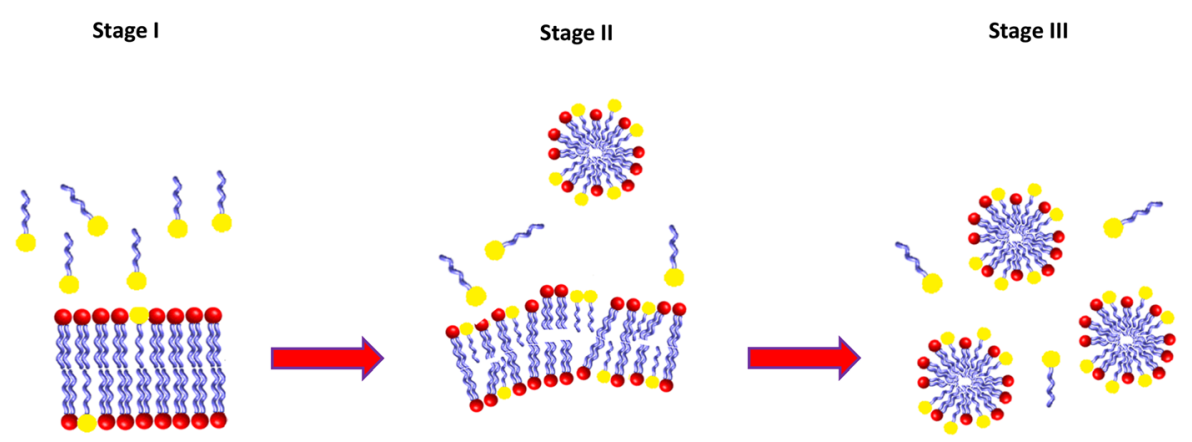

Fig. 3 Surfactants-lipids interaction: the three-stage model. Stage I: Surfactant molecules approach a bilayer. Stage II: Combination of micelles and lipid/surfactant aggregates. Stage III: Mixed micelles formation 
(Cui et al. 2010; Hosseinkhani et al. 2013; Dehsorkhi et al. 2014; Du and Stenzel 2014; Malaspina et al. 2017; Doostmohammadi et al. 2019).

An important class of amphiphilic peptides called surfactant-like peptides (SLPs), present an intrinsic difference that can lead to different physical consequences namely composition and tail structure (Malaspina et al. 2017). Unlike conventional surfactants whose hydrophobic tails interact in all directions through hydrophobic interactions, the amphiphilic peptide tail contains not only hydrophobic groups but also hydrophilic sites (Colherinhas and Fileti 2014). This feature allows the SLPs to stabilize nanostructures in one direction through hydrophobic interactions and in the orthogonal direction by hydrogen bonds. These hydrogen bonds associated with hydrophobic interactions can stabilize at a high level, complex secondary structures such as helices and sheets. On the other hand, conventional lipids/surfactants with antimicrobials properties (Chen et al. 2010, 2012; Albada et al. 2012; Gaspar et al. 2013) are usually organized into micelles, vesicles, and nanotubes (Colherinhas and Fileti 2014; Malaspina et al. 2017). To understand the interaction between (bio)surfactants and lipids, it is necessary to be aware of the hydrodynamics of the molecules involved, their amphiphilic properties and how they play an important role when it comes to biological membranes. Nanoparticle models and the study of their properties could help us to understand the molecular basis of these interactions, which have remained unknown.

\section{Surfactant-polysaccharide interactions}

Polysaccharides are monosaccharide (homo or hetero) built up biopolymers mainly produced by plants. Similar to surfactants, they could be classified based on their charge as non-ionic (o), cationic (+), and anionic (-) polymers (Kwak 1998). Polysaccharides and surfactant interactions are important to develop (a) emulsifiers; (b) flocculating agents; (c) stabilizing colloids; (d) or rheology controllers (Holmberg et al. 2002) in food, medicine and environmental applications.

Electrostatic, hydrophobic, dipole-dipole, and hydrogen bonding interactions along with the surfactant and polysaccharide characteristic are the main factors that affect the Polymer-Surfactant Systems
(PSS) (Grządka et al. 2019). These interactions have been summarised in Table 1 (Bao et al. 2008). These authors studied the interactions of ionic surfactants (SDS and CTAB) with neutral, positively, and negatively charged polysaccharides [Methyl cellulose (MC), chitosan (CS) and $\kappa$-carrageenan (KC)], respectively.

According to the surfactant-polysaccharide combination, molecular interactions change. Therefore, strong hydrophobic and weak ion-dipole interactions are present in MC-SDS mixture. Moreover, in $\mathrm{KC}-$ SDS and CS-SDS, ionic interactions drive the binding process between surfactant and the polymer. Hydrophobic interactions are weak in KC-SDS, while in CS-SDS, polymer hydrophobic moieties interact with alkyl chains of the SDS. In the case of CTAB with $\mathrm{MC}$ and $\mathrm{CS}$, only hydrophobic interactions are present, and strong electrostatic interactions allow binding between $\mathrm{KC}$ and CTAB.

In the case of non-ionic polysaccharide and anionic surfactant, as ethyl hydroxyethyl cellulose (EHEC) and SDS, respectively, the hydrophobic interaction between the polymer and SDS alkyl chain drives their association. Accordingly, SDS plays an important role because its presence or absence promotes the extent of EHEC-SDS cluster formation. For example, if SDS concentration is below the critical aggregation concentration (CAC), surface tension is reduced depending on SDS molecules, but when SDS concentration increases to at or above the CAC, EHEC adsorption is accelerated. In diluted solutions, the surface activity is strong (12 ppm of EHEC and $2 \mathrm{mM}$ SDS), making this PSS a vehicle for drug delivery (Nahringbauer 1997).

Cationic surfactants such as DTAB, MTAB, and $\mathrm{CTAB}$, interact with cellulose in the water interface. These cationic surfactants contain a different number of $-\mathrm{CH}_{2}-$ groups in the alkyl chain, and their $\mathrm{CMC}$ varies with respect to alkyl chain length $(\mathrm{CTAB}>$ MTAB $>$ DTAB). The chain length of this kind of cationic surfactants influences interaction behaviour with non-ionic polysaccharides such as cellulose. For example, CTAB-cellulose interaction is driven by hydrophobic interactions, while electrostatic interactions are very significant in interactions of MTAB and DTAB with cellulose, respectively.

In the case of interactions of polysaccharides such as dextrin and carboxymethylcellulose with cationic surfactant groups (DTAB, MTAB, CTAB), the 
Table 1 Interactions between methyl cellulose, chitosan and $\kappa$-carrageenan with ionic surfactants, SDS and CTAB (Bao et al. 2008)

\begin{tabular}{lllll}
\hline Polysaccharide & Surfactant & Interaction & & \\
\cline { 3 - 5 } & & Hydrophobic & Electrostatic & Ion-dipole \\
\hline Methyl cellulose & SDS & Strong & & Weak \\
$\begin{array}{l}\text { Chitosan } \\
\kappa-\text { Carrageenan }\end{array}$ & & Medium & Strong & \\
$\begin{array}{l}\text { Methyl cellulose } \\
\text { Chitosan }\end{array}$ & CTAB & Weak & & \\
$\kappa$-Carrageenan & & Strong & & \\
\hline
\end{tabular}

Table 2 Interaction of cetyl trimethyl ammonium bromide family $(\mathrm{CnTAB})$ with cellulose nanocrystals $(\mathrm{C}=$ carbon number in alkyl chain of surfactant) (Brinatti et al. 2016)

\begin{tabular}{llll}
\hline & CnTAB interaction with cellulose nanocrystals & Micelle formation & Flocculation \\
\hline $\mathrm{C}=12$ & Electrostatic & High concentration & High concentration \\
$\mathrm{C}=14$ & Electrostatic-hydrophobic & Low concentration & High concentration \\
$\mathrm{C}=16$ & Electrostatic-hydrophobic & Low concentration & . \\
\hline
\end{tabular}

behaviour is different, for the interaction between dextrin and CTAB, hydrophobicity drives the interaction while in the case of carboxymethylcellulose and $\mathrm{CTAB}$, electrostatic interactions are very significant (Biswas and Chattoraj 1997a, b).

Another example of PSS with an anionic surfactant, sodium stearoyl lactylate (SSL), an anionic surfactant and $\kappa$-carrageenan $(\mathrm{KC})$ polymer, both of which are important in the food industry, have a different behaviour in solutions and gels. SSL changes $\mathrm{KC}$ conformation due to electrostatic interactions and hindrance. In the gelation process (melting process), KC suffers a coil helix transition and, finally, helixhelix aggregation, modifying its melting enthalpy. SSL hinders KC helix-helix aggregation. But, at a high concentration of surfactant, SSL forms micelles (solutions and gels). The combination of hindrance and electrostatic repulsion promote conformational changes in $\mathrm{KC}$, both in solutions and in gels. In solution, enthalpy decreases continuously at high SSL concentration range, while in gels, this parameter decreases at a specific SSL concentration (OrtizTafoya et al. 2018).

In other cases, the interaction between a polysaccharide and surfactant depends on the alkyl chain length of the tensioactive molecule. Such is the case of CTAB homologues (CnTAB, where $\mathbf{n}$ is a carbon number in alkyl chain of surfactant) with cellulose nanocrystals, a negatively charged polysaccharide. When $\mathbf{n}=12$ and the surfactant concentration is high, electrostatic interactions are present and micelle formation occurs, while at $\mathbf{n}=14-16$ and a low surfactant concentration, micelles are formed, and flocculation process occurs at high CnTAB concentration (Table 2). These processes are driven in first instance by electrostatic interactions and by the hydrophobic interactions (Brinatti et al. 2016).

\section{Polysaccharides-biosurfactants interactions}

Some biosurfactants contain sugars in their structure such as glycolipids (e.g. rhamnolipids) and also interact with polysaccharides. In the food and pharmaceutical industries, pickering/stabilizing high internal phase emulsions (HIPEs) are very important as they are used in bioactive delivery. In these HIPEs three kinds of molecules interact: proteins-polysaccharides-biosurfactants. For example, zein-propylene glycol alginate mixed with rhamnolipids stabilize pickering emulsion in the oil-in-water interface. This emulsion system is formed by a 3D network of adsorbed and non-adsorbed particles, however the basis of molecular interactions amongst these molecules is unclear (Dai et al. 2019). 


\section{Conclusions}

Recent works in this area highlight the importance of the interactions between surfactants and macromolecules and their role in biological membranes. The structures that form in solution are driven by molecular interactions. There are three main forces that drive the protein-surfactant interactions: electrostatic, hydrophobic, and Van der Waals, while the dominant interaction is controlled by the characteristics of both molecules and their concentration. The interactions between lipids and surfactants are described as a three-stage model, starting with the surfactant partition between the lipid bilayers and the aqueous phase, reaching a level where the bilayers break into micelles and ending with the solubilization of bilayers. The characteristics of PSS, such as polysaccharides-surfactants, can be controlled through the molecule design and their charge i.e. presence of electrostatic interaction at opposite charge PPS where hydrophobic interactions are predominant in $\mathrm{o} /-$ and $\mathrm{o} /+\mathrm{PPS}$, and where $\mathrm{o} /-$ interaction is stronger than $\mathrm{o} /+\mathrm{PSS}$; these are some of the most powerful parameters to take into account in order to obtain the desired structures to be used for different applications. By modifying the interaction type and strength, as well as the concentrations of the molecules involved, the final product can be used for a wide variety of industrial formulations.

Acknowledgements The authors acknowledge the funding support from the Faculty of Science, Liverpool John Moores University ECR Fellowship 2018-2019. We also acknowledge to CAC Biología Celular y Molecular, Universidad Autónoma de Ciudad Juárez for its host facilities.

Open Access This article is licensed under a Creative Commons Attribution 4.0 International License, which permits use, sharing, adaptation, distribution and reproduction in any medium or format, as long as you give appropriate credit to the original author(s) and the source, provide a link to the Creative Commons licence, and indicate if changes were made. The images or other third party material in this article are included in the article's Creative Commons licence, unless indicated otherwise in a credit line to the material. If material is not included in the article's Creative Commons licence and your intended use is not permitted by statutory regulation or exceeds the permitted use, you will need to obtain permission directly from the copyright holder. To view a copy of this licence, visit http://creativecommons.org/licenses/by/4.0/.

\section{References}

Afinjuomo F, Fouladian P, Parikh A et al (2019) Preparation and characterization of oxidized inulin hydrogel for controlled drug delivery. Pharmaceutics 11:356

Akbari S, Abdurahman NH, Yunus RM et al (2018) Biosurfactants-a new frontier for social and environmental safety: a mini review. Biotechnol Res Innov 2(1):81-90

Akram M, Ansari F, Bhat IA et al (2019) Probing interactions of bovine serum albumin (BSA) with the biodegradable version of cationic gemini surfactant. J Mol Liq 276:519-528

Albada HB, Prochnow P, Bobersky S et al (2012) Tuning the activity of a short Arg-Trp antimicrobial peptide by lipidation of a C-or N-terminal lysine side-chain. ACS Med Chem Lett 3(12):980-984

Aranda FJ, Espuny MJ, Marqués A et al (2007) Thermodynamics of the interaction of a dirhamnolipid biosurfactant secreted by Pseudomonas aeruginosa with phospholipid membranes. Langmuir 23:2700-2705

Asselin S, Ingram J (2014) Uranium leaching from contaminated soil utilizing rhamnolipid, EDTA, and citric acid. Appl Environ Soil Sci 2014:462514

Banat IM, Thavasi R (2018) Microbial biosurfactants and their environmental and industrial applications. CRC Press, Boca Raton, USA

Bao H, Lin L, Gan LH et al (2008) Interactions between ionic surfactants and polysaccharides in aqueous solutions. Macromolecules 41:9406-9412

Behrens R (1964) The physical and chemical properties of surfactants and their effects on formulated herbicides. Weeds 12:255

Biswas SC, Chattoraj DK (1997a) Polysaccharide-surfactant interaction. 1. Adsorption of cationic surfactants at the cellulose-water interface. Langmuir 13:4505-4511

Biswas SC, Chattoraj DK (1997b) Polysaccharide-surfactant interaction. 2. Binding of cationic surfactants to carboxymethyl cellulose and dextrin. Langmuir 13:4512-4519

Bnyan R, Khan I, Ehtezazi T et al (2018) Surfactant effects on lipid-based vesicles properties. J Pharm Sci 107(5):1237-1246

Brinatti C, Huang J, Berry RM et al (2016) Structural and energetic studies on the interaction of cationic surfactants and cellulose nanocrystals. Langmuir 32:689-698

Campos JM, Montenegro Stamford TL, Sarubbo LA, de Luna JM, Rufino RD, Banat IM (2013) Microbial biosurfactants as additives for food industries; a review. Biotechnol Prog 29(5):1097-1108

Castelli F, Sarpietro MG, Micieli D et al (2008) Differential scanning calorimetry study on drug release from an inulinbased hydrogel and its interaction with a biomembrane model: $\mathrm{pH}$ and loading effect. Eur $\mathrm{J}$ Pharm Sci 35(1-2):76-85

Ceresa C, Fracchia L, Williams M, Banat IM et al (2020) The effect of sophorolipids against microbial biofilms on medical-grade silicone. J Biotechnol 309:34-43

Chen C, Pan F, Zhang S, Hu J et al (2010) Antibacterial activities of short designer peptides: a link between propensity for nanostructuring and capacity for membrane destabilization. Biomacromolecules 11(2):402-411 
Chen C, Hu J, Zhang S et al (2012) Molecular mechanisms of antibacterial and antitumor actions of designed surfactantlike peptides. Biomaterials 33(2):592-603

Chernik GG (2000) Phase studies of surfactants-water systems. Curr Opin Colloid Interface Sci 4:381-390

Colherinhas G, Fileti E (2014) Molecular dynamics study of surfactant-like peptide based nanostructures. J Phys Chem B 118(42):12215-12222

Cui H, Webber M, Stupp SI (2010) Self-assembly of peptide amphiphiles: from molecules to nanostructures to biomaterials. Biopolymers 94(1):1-18

Dai L, Yang S, Wei Y et al (2019) Development of stable high internal phase emulsions by pickering stabilizations: utilization of zein-propylene glycol alginate-rhamnolipid complex particles as colloidal emulsifiers. Food Chem 275:246-254

Deep S, Ahluwalia JC (2001) Interaction of bovine serum albumin with anionic surfactants. Phys Chem Chem Phys 3:4583-4591

Dehsorkhi A, Castelletto V, Hamley IW (2014) Self-assembling amphiphilic peptides. J Pept Sci 20(7):453-467

Díaz X, Abuin E, Lissi E (2003) Quenching of BSA intrinsic fluorescence by alkylpyridinium cations: its relationship to surfactant-protein association. J Photochem Photobiol 155:157-162

Diaz MA, De Ranson IU, Dorta B et al (2015) Metal removal from contaminated soils through bioleaching with oxidizing bacteria and rhamnolipid biosurfactants. Soil Sediment Contam Int J 24:16

Diaz De Rienzo MA, Kamalanathan ID, Martin PJ (2016) Comparative study of the production of rhamnolipid biosurfactants by $B$. thailandensis E264 and $P$. aeruginosa ATCC 9027 using foam fractionation. Process Biochem 51:820-827

Doostmohammadi M, Ameri A, Mohammadinejad R, Banat IM, Ohadi M, Dehghannoudeh G (2019) Hydrogels for peptide hormones delivery: therapeutic and tissue engineering applications. Drug Des Dev Ther 13:3405-3418

Du AW, Stenzel MH (2014) Drug carriers for the delivery of therapeutic peptides. Biomacromolecules 15(4):1097-1114

Dua JS, Rana AC, Bhandari AK (2012) Liposome: methods of preparation and applications. Int $\mathbf{J}$ Pharm Stud Res 3(2): 14-20

Dubey P, Sugam K, Ravindranathan S et al (2018) pH dependent sophorilipid assemblies and their influence on gelation of silk fibrion protein. Mater Chem Phys 23:9-16

Ebnesajjad S (2006) Surface treatment of materials for adhesive bonding. In: Ebnesajjad S (ed) Surface tension and its measurement, 2nd edn. Elsevier, USA, pp 9-28

Ebnesajjad S (2014) Surface treatment of materials for adhesive bonding. In: Ebnesajjad S (ed) Surface tension and its measurement, 2nd edn. Elsevier, USA, pp 7-24

Elshikh M, Moya-Ramírez I, Moens H et al (2017) Rhamnolipids and lactonic sophorolipids: natural antimicrobial surfactants for oral hygiene. J Appl Microbiol 123:1111-1123

Fan L, Xie P, Wang Y et al (2018) Biosurfactant-protein interaction: influences of mannosylerythritol lipids-A on $\beta$ glucosidase. J Agric Food Chem 66:238-246
Faustino CMC, Calado ART, García-Rio L (2009) Geminisurfactant-protein interactions: effect of $\mathrm{pH}$, temperature, and surfactant stereochemistry. Biomacromolecules 10:2508-2514

Gaspar D, Veiga AS, Castanho MARB (2013) From antimicrobial to anticancer peptides. A review. Front Microbiol 4:294

Geetha SJ, Banat IM, Joshi SJ (2018) Biosurfactants: production and potential applications in microbial enhanced oil recovery (MEOR). Biocatal Agric Biotechnol 14:23-32

Grządka E, Matusiak J, Stankeviĉ M (2019) Interactions between fluorocarbon surfactants and polysaccharides. J Mol Liq 283:81-90

Gunay SM, Ozer Y (2018) Liposomes and micelles as nanocarriers for diagnostic and imaging purposes In: Design of nanostructures for theranostics applications. William Andrew Publishing, USA

Hansted JG, Wejse PL, Bertelsen H et al (2011) Effect of protein-surfactant interactions on aggregation of $\beta$-lactoglobulin. Biochim Biophys Acta 1814:713-723

Helenius A, Simons K (1975) Solubilization of membranes by detergents. Biochim Biophys Acta 415:29-79

Holmberg K, Joensson B, Kronberg B et al (2002) Surfactants and polymers in aqueous solution. Wiley, Chichester, UK

Hosseinkhani H, Hong PD, Yu DS (2013) Self-assembled proteins and peptides for regenerative medicine. Chem Rev 113:4837-4861

Kezwon A, Góral I, Fraczyk T et al (2016) Effect of surfactants on surface activity and rheological properties of type I collagen at air/water interface. Colloid Surf B 148:238-248

Kezwoń A, Wojciechowski K (2016) Collagen-surfactant mixtures at fluid/fluid interfaces. Colloid Surf A 509:390-400

Khan TA, Mahler HC, Kishore RSK (2015) Key interactions of surfactants in therapeutic protein formulations: a review. Eur J Pharm Biopharm 97:60-67

Khan JM, Malik A, Ahmed A et al (2019) Effect of cetyltrimethylammonium bromide (CTAB) on the conformation of a hen egg white lysozyme: a spectroscopic and molecular docking study. Spectrochim Acta A Mol Biomol Spectrosc 219:313-318

Koynova R, Tenchov B (2001) Interactions of surfactants and fatty acids with lipids. Curr Opin Colloid Interface Sci 6:277-286

Kralova I, Sjöblom J (2009) Surfactants used in food industry: a review. J Dispers Sci Technol 30(9):1363-1383

Kwak JCT (1998) Polymer-surfactant systems. Marcel Dekker, USA

Langner M, Hui S (2000) Effect of free fatty acids on the permeability of 1,2-dimyristoyl-sn-glycero-3-phosphocholine bilayer at the main phase transition. Biochim Biophys Acta 1463(2):439-447

Le Maire M, Champeil P, Moller JV (2000) Interaction of membrane proteins and lipids with solubilizing detergents. Biochim Biophys Acta 1508:86-111

Lee HJ, McAuley A, Schilke KF et al (2011) Molecular origins of surfactant-mediated stabilizations of protein drugs. Adv Drug Deliv Rev 63:1160-1171

Li Y, Lee JS (2019) Staring at protein-surfactant interactions: fundamental approaches and comparative evaluation of their combinations: a review. Anal Chim Acta 1063:18-39 
Lichtenberg D, Robson RJ, Dennis EAW (1983) Solubilization of phospholipid by detergents structural and kinetic aspects. Biochim Biophys Acta 737:285-304

Lichtenberg D, Ahyayauch H, Alonso A et al (2013) Detergent solubilization of lipid bilayers: a balance of driving forces. Trends Biochem Sci 38(2):85-93

Mackie A, Wilde P (2005) The role of interactions in defining the structure of mixed protein-surfactant interfaces. Adv Colloid Interface Sci 117:3-13

Malaspina T, Colherinhas G, Outi FO et al (2017) Assessing the interaction between surfactant-like peptides and lipid membranes. RSC Adv 7:35973-35981

Maldonado F, Almela M, Otero A et al (1991) The binding of anionic and nonionic surfactants to collagen through the hydrophobic effect. J Protein Chem 10(2):189-192

Malik NA (2015) Surfactant-amino acid and surfactant-surfactant interaction in aqueous medium: a review. Appl Biochem Biotechnol 176:2077-2106

Mandal BB, Kund SC (2008) A novel method for dissolution and stabilization of non-mulberry silk gland protein fibroin using anionic surfactant sodium dodecyl sulfate. Biotechnol Bioeng 99:1482-1489

Mankowich AM (1953) Physicochemical properties of surfactants. Ind Eng Chem 45(12):2759-2766

Marquez R, Forgiarini AM, Langevin D et al (2018) Instability of emulsions made with surfactant-oil-water systems at optimum formulation with ultralow interfacial tension. Langmuir 34:9252-9263

Marquez R, Anton R, Vejar F et al (2019) New interfacial rheology characteristics measured using a spinning drop Rheometer at the optimum formulation. Part 2. Surfactantoil-water systems with a high volume of middle-phase microemulsion. J Surfactants Deterg 22:177-188

Mehan S, Aswal VK, Kohlbrecher J (2015) Tuning of proteinsurfactant interaction to modify the resultant structure. Phys Rev E 92:032713

Morais IMC, Cordeiro AL, Teixeira GS et al (2017) Biological and physicochemical properties of biosurfactants produced by Lactobacillus jensenii P6A and Lactobacillus gasseri P65. Microb Cell Fact 16(155):1-15

Nahringbauer I (1997) Polymer-surfactant interaction as revealed by the time dependence of surface tension. The EHEC/SDS/water system. Langmuir 13:2242-2249

Naughton PJ, Marchant R, Naughton V et al (2019) Microbial biosurfactants: current trends and applications in agricultural and biomedical industries. J Appl Microbiol 127:12-28

Ohadi M, Shahravan A, Dehghannoudeh N, Eslaminejad T, Banat IM, Dehghannoudeh G (2020) Potential use of microbial surfactant in microemulsion drug delivery system: a systematic review. Drug Des Dev Ther 14:541-550

Ortiz A, Teruel JA, Espuny MJ et al (2008) Interactions of a Rhodococcus sp. biosurfactant trehalose lipid with phosphatidylethanolamine membranes. Biochim Biophys Acta 1778:2806-2813

Ortiz A, Teruel JA, Espuny MJ et al (2009) Interactions of a bacterial biosurfactant trehalose lipid with phosphatidylserine membranes. Chem Phys Lipids 158(1):46-53

Ortiz-Tafoya MC, Rolland-Sabaté A, Garnier C et al (2018) Thermal, conformational and rheological properties of $\kappa$ - carrageenan-sodium stearoyl lactylate gels and solutions. Carbohydr Polym 193:289-297

Otzen D (2011) Protein-surfactant interactions: a tale of many states. Biochim Biophys Acta 1814:562-591

Otzen DE (2017) Biosurfactants and surfactants interacting with membranes and proteins: same but different? Biochim Biophys Acta 1859:639-649

Otzen DE, Sehgal P, Westh P (2009) $\alpha$-Lactalbumin is unfolded by all classes of surfactants but by different mechanisms. J Colloid Interface Sci 329:273-283

Pacifico C, Giers S (1995) Surfactants used in textile applications. J Am Oil Chem Soc 32:231-235

Pan F, Lu Z, Tucker I et al (2016) Surface active complexes formed between keratin polypeptides and ionic surfactants. J Colloid Interface Sci 484:125-134

Park JH, Kim MH, Jeong L et al (2014) Effect of surfactants on sol-gel transition of silk fibroin. J Sol-Gel Sci Technol 71:364-371

Parray M, Mir MUH, Dohare N et al (2018) Effect of cationic gemini surfactant and its monomeric counterpart on the conformational stability and esterase activity of human serum albumin. J Mol Liq 260:65-77

Patino JMR, Niño MRR, Sánchez CC (2007) Physico-chemical properties of surfactant and protein films. Curr Opin Colloid Interface Sci 12(4):187-195

Peypoux F, Bonmatin JM, Labbe H et al (1994) [Ala4] surfactin, a novel isoform from $B$. subtilis studied by mass and NMR spectroscopies. Eur J Biochem 224:89-96

Pizzirusso A, De Nicola A, Sevink GJA et al (2017) Biomembrane solubilization mechanism by Triton X-100: a computational study of the three stage model. Phys Chem Chem Phys 19:29780-29794

Poša M, Pilipović A, Torović L et al (2019) Co-solubilisation of a binary mixture of isoflavones in a water micellar solution of sodium cholate or cetyltrimethylammonium bromide: influence of micelle structure. J Mol Liq 273:134-146

Proffitt TJ, Patterson T (1988) Oleochemical surfactants and lubricants in the textile industry. J Am Oil Chem Soc 65:1682

Rodrigues L, Banat IM, Teixeira J et al (2006) Biosurfactants: potential applications in medicine. J Antimicrob Chemother 57:609-618

Rudolph TW, Jones LS (2002) Surfactant-protein interactions. Pharm Biotechnol 13:159-175

Ruiz-Peña M, Oropesa-Nuñez TP, Louro SRW et al (2010) Physico-chemical studies of molecular interactions between non-ionic surfactants and bovine serum albumin. Colloids Surf B Biointerfaces 75:282-289

Santos D, Rufino R, Luna J et al (2016) Biosurfactants: multifunctional biomolecules of the 21 st century. Int J Mol Sci 17:401

Sinha S, Tikariha D, Lakra J et al (2016) Interactions of bovine serum albumin with cationic monomeric and dimeric surfactants: a comparative study. J Mol Liq 218:421-428

Sonu SH, Kumari S, Aggrawal R et al (2017) Study on interactions of cationic gemini surfactants with folded and unfolded bovine serum albumin: effect of spacer group of surfactants. J Mol Liq 245:369-379

Stryer S (1981) Kimball's biology pages, "cell membranes." Biochemistry 2nd ed. Freeman, USA 
Tucker IA, Petkov JT, Penfold J et al (2014) Spontaneous surface self-assembly in protein-surfactant mixtures: interactions between hydrophobin and ethoxylated polysorbate surfactants. J Phys Chem 118:4867-4875

Thimon L, Peypoux F, Wallach J et al (1995) Effect of lipopeptide antibiotic, iturin A, on morphology and membrane ultrastructure of yeast cells. FEMS Microbiol Lett 128:101-106

Van Os NM, Haak JR, Rupert LAM (1993) Physico-chemical properties of selected anionic, cationic and nonionic surfactants. Elsevier, USA
Zaragoza A, Teruel JA, Aranda FJ et al (2012) Interaction of a Rhodococcus sp. trehalose lipid biosurfactant with model proteins: thermodynamic and structural changes. Langmuir 28(2):1381-1390

Zhang J, Li Y (2018) Study on the interaction between surfactin and alkaline protease in aqueous solution. Int $\mathrm{J}$ Biol Macromol 118:244-251

Publisher's Note Springer Nature remains neutral with regard to jurisdictional claims in published maps and institutional affiliations. 